1 Hacettepe Journal of Mathematics and Statistics

$\bigcap$ Volume 48 (4) (2019), 1213-1231

RESEARCH ARTICLE

\title{
Panel data unit root test with structural break: A Bayesian approach
}

\author{
Jitendra Kumar*广 (1) and Varun Agiwal ${ }^{\ddagger}$ (1)
}

\begin{abstract}
The idea about structural break in unit root hypothesis under time series model had received great amount of attention over many last decades. The importance of structural break in the mean had been comprehensively studied by Perron [15], Perron and Vogelsang [17], Zivot and Andrews [25] etc. This had also studied in considering of break in variance by Kim et al. [9], Cook [6], Kumar et al. [11] etc. There is sufficient contribution regarding break in mean and variance individually but both are equally important and this was little explored by Bai [1] for panel data and Meligkotsidou et al. [14] for univariate time series. In present paper, we are extending this on panel data $\mathrm{AR}(1)$ time series model under Bayesian framework. Posterior odds ratio has been derived for various models with and without break in mean, variance and both in consideration of unit root hypothesis. A simulation as well as an empirical analysis is also carried out to get more generalized view on the model under study.
\end{abstract}

Keywords: Panel autoregressive model, Structural break, Prior distribution, Posterior odds ratio, Monte Carlo integration.

Mathematics Subject Classification (2010): 62F15, 37M10, 65C05

Received: 19.02.2018 Accepted : 19.09.2018 Doi : 10.15672/HJMS.2018.626

${ }^{*}$ Department of Statistics, Central University of Rajasthan, Bandersindri, Ajmer, India, Email: jitendravkarma@curaj.ac.in

$\dagger$ Corresponding Author.

${ }_{\ddagger}^{\ddagger}$ Department of Statistics, Central University of Rajasthan, Bandersindri, Ajmer, India, Email: varunagiwal.stats@gmail.com 


\section{Introduction}

A collection of time dependent set of observations recorded in order to its occurrence is called time series. Time series data are analyzed to identify the patterns or structure of data for understanding and modeling to have better prediction. This is not very easy because each observation is dependent upon previous observation with respect to their particular time see Box and Jenkins [3]. The structure of time series data is affected by several reasons and changing the trend of the series. Such type of change are called structural break.

The problem of structural breaks in any level such as mean/slope shifting of a series has established significant attention in association with unit root hypothesis. Yao [23] studied the estimation and testing the number of shifts in the mean variable using Bayesian information criterion. Perron and Vogelsang [17] and Perron [16] proposed a class of test statistics for two different forms of structural break which allow the changes in both level and trend. However, relative to the classical literature on unit root against structural break determination, structural breaks are less explored under Bayesian approach in comparison to classical approach. Chib [5] had developed a general approach for estimation multiple change points via Monte Carlo Markov Chain (MCMC) algorithm. Chaturvedi and Kumar [4] proposed posterior odds ratio for unit root hypothesis under the condition in which break are present in mean and trend component for $\mathrm{AR}(1)$ time series model and it is further extended for multiple structural breaks. Shao and Zhang [20] proposed a self-normalization (SN) based Kolmogorov-Smirnov test for testing the mean shift in univariate time series and extended for multiple change points.

Sometimes variance associated with the error term is also affect the structure of model and if not taken into account then give misleading conclusion. Inclan [7] suggested a method to find the break point in variance using Bayesian method. Wang and Zivot [22] started with a deterministically trending dynamic time series model in which multiple structural changes in level, trend, and error variance are modeled and then estimated the parameter using Gibbs sampling. Bai [1] obtained the consistency on estimated common break point in panel data and used least squares method for estimating breaks in mean and the quasi-maximum likelihood (QML) method to estimate break in both mean and variance. Using Bayesian approach, Meligkotsidou et al. [14] considered structural change in level, error variance and autoregressive coefficient at unknown break point and estimation the model parameters by using marginal likelihood. Kumar et al. [11] investigated the impact of structural break in error variance using Bayesian framework and applied on export data of selected ASEAN countries for AR(1) time series model .

Recently work had been done in time series and econometric in reference of panel data time series model considering structural break by few researcher. Li et al. [13] introduced a penalized principal component (PPC) estimation procedure to detecting the multiple structural breaks in the panel data models with unobservable interactive fixed effects and show that unobservable and unknown number of structural breaks can be consistently estimated. Qian and Su [18] studied the estimation technique and inference of common breaks in panel data models with and without interactive fixed effects using LASSOtype methods. Baltagi et al. [2] worked on common correlated effects (CCE) estimators for large heterogeneous panels with a general multi factor error structure with unknown structural breaks and Monte Carlo simulations was used to verify the consistency of change point for correlated effects model. Kim et al. [10] discussed the wild bootstrap method and apply Leybourne-Kim-Newbold unit root test in the presence of single or multiple breaks in variance.

There are sufficient works on unit root hypothesis for a panel data time series model but it is less explored in case of structural break specially considering break in both. 
The present paper dealt structural break in both mean and variance under Bayesian framework and explored various hypothesis under different setup such as break in both mean and variance, break in variance, break in mean, no break in association with unit root hypothesis. Thus, one can be tested or analyze one versus other hypothesis by using posterior odds ratio i.e. different combination of null $\left(H_{0}\right)$ against alternative $\left(H_{a}\right)$ hypothesis can be tested. The proposed analysis is verified by the simulation as well as empirical study and gets sufficient scope to justify the present study.

\section{Model with Structural Breaks in Mean and Variance}

Let a panel data time series $\left\{y_{i t} ; i=1,2, \ldots, n ; t=1,2, \ldots, T\right\}$ follows the time series model with structural break where break is present at a single point time $T_{B}$

$$
y_{i t}= \begin{cases}\mu_{i 1}+u_{i t} & \text { for } t=1,2, \ldots, T_{B} \\ \mu_{i 2}+u_{i t} & \text { for } t=T_{B}+1, \ldots, T\end{cases}
$$

where stochastic error term $u_{i t}$ follows panel $A R(1)$ process and are i.i.d. random variables follows $N\left(0, \tau^{-1}\right)$ as given below

$$
u_{i t}= \begin{cases}u_{i, t-1}+\varepsilon_{i t} & \text { for } t=1,2, \ldots, T_{B} \\ u_{i, t-1}+\lambda \varepsilon_{i t} & \text { for } t=T_{B}+1, \ldots, T\end{cases}
$$

We may write equation (2.1) by using (2.2)

$$
y_{i t}= \begin{cases}\rho y_{i t-1}+(1-\rho) \mu_{i 1}+\varepsilon_{i t} & \text { for } t=1,2, \ldots, T_{B} \\ \rho y_{i t-1}+(1-\rho) \mu_{i 2}+\lambda \varepsilon_{i t} & \text { for } t=T_{B}+1, \ldots, T\end{cases}
$$

The model defined in (2.3) is a panel AR(1) model when structural break occurs on both mean and variance. Our interest is to test the hypothesis that model is stationary or not in association with structural break. Model (2.3) can be explored for all possible particular form of model which are listed below:

(1) Model is trend stationary and break occurs on mean as well as variance in such case series follows model (2.3) and equivalently hypothesis is $H^{1}: \rho \epsilon s, \mu_{i 1} \neq$ $\mu_{i 2}, \lambda \neq 1, s=\{a: a<\rho<1 ; a>-1\}$

(2) Model is difference stationary and break occurs on both mean (intercept) as well as variance. However break in mean is not impact in this case. Equivalently, hypothesis is $H^{2}: \rho=1, \mu_{i 1} \neq \mu_{i 2}, \lambda \neq 1$ or $H^{3}: \rho=1, \mu_{i 1}=\mu_{i 2}, \lambda \neq 1$ and model (2.3) reduces to

$$
\Delta y_{i t}= \begin{cases}\varepsilon_{i t} & \text { for } t=1,2, \ldots, T_{B} \\ \lambda \varepsilon_{i t} & \text { for } t=T_{B}+1, \ldots, T\end{cases}
$$

(3) Model is trend stationary and break occurs only is variance equivalently hypothesis is $H^{4}: \rho \epsilon s, \mu_{i 1}=\mu_{i 2}=\mu_{i}($ say $), \lambda \neq 1, s=\{a: a<\rho<1 ; a>-1\}$ is similar hypothesis given by (Kumar et al. 2012) for univariate case and model follows:

$$
y_{i t}= \begin{cases}\rho y_{i t-1}+(1-\rho) \mu_{i}+\varepsilon_{i t} & \text { for } t=1,2, \ldots, T_{B} \\ \rho y_{i t-1}+(1-\rho) \mu_{i}+\lambda \varepsilon_{i t} & \text { for } t=T_{B}+1, \ldots, T\end{cases}
$$

(4) Model is trend stationary and break occurs only with mean i.e. intercept which is similar hypothesis as (Perron 1990). Equivalently hypothesis is $H^{5}: \rho \epsilon s, \mu_{i 1} \neq$ $\mu_{i 2}, \lambda=1, s=\{a: a<\rho<1 ; a>-1\}$ and (2.3) reduces in the form

$$
y_{i t}= \begin{cases}\rho y_{i t-1}+(1-\rho) \mu_{i 1}+\varepsilon_{i t} & \text { for } t=1,2, \ldots, T_{B} \\ \rho y_{i t-1}+(1-\rho) \mu_{i 2}+\varepsilon_{i t} & \text { for } t=T_{B}+1, \ldots, T\end{cases}
$$


(5) Model is difference stationary and break occurs only with mean i.e. intercept but in case of difference stationary mean term is vanishes, therefore model is free from the impact of break in mean. This is similar null hypothesis taken by (Levin et al. 2002). Equivalently hypothesis is consider $H^{6}: \rho=1, \mu_{i 1} \neq \mu_{i 2}, \lambda=1$ or $H^{7}: \rho=1, \mu_{i 1}=\mu_{i 2}, \lambda=1$ and model (2.3) is reduces to

$\Delta y_{i t}=\varepsilon_{i t}$ for $t=1,2, \ldots, T$

(6) Series is trend stationary and no break occurs, this model is initially explored for the unit root hypothesis consider by (Levin et al. 2002). Equivalently hypothesis is $H^{8}: \rho \in s, \mu_{i 1}=\mu_{i 2}=\mu_{i}($ say $), \lambda=1, s=\{a: a<\rho<1 ; a>-1\}$ and the model come out is

$$
y_{i t}=\rho y_{i t-1}+(1-\rho) \mu_{i}+\varepsilon_{i t} \text { for } t=1,2, \ldots, T
$$

\section{Prior Distributions}

In Bayesian for making inference need some specify prior distribution for the model parameters. Here, we considered both informative and non-informative priors for the unknown parameters. Let us assume the following prior distributions for the parameters used in the model (Schotman and Van Dijk [19]).

$$
\begin{aligned}
& \mu_{i j} \sim N\left(y_{i 0}, \frac{1}{\tau\left(1-\rho^{2}\right)}\right) ; j=1,2 \\
& p(\tau) \propto \frac{1}{\tau} ; \quad 0<\tau<\infty \\
& p(\lambda) \propto \frac{1}{\lambda} ; \quad 0<\lambda<\infty \\
& p(\rho) \propto \frac{1}{1-a} ; \quad a<\rho<1, a>-1 \\
& O\left(H_{0}\right)=\frac{\theta}{1-\theta}
\end{aligned}
$$

The joint prior distribution is given by

$$
\begin{aligned}
\pi(\Theta) & =\pi\left(\mu_{i 1}\right) \pi\left(\mu_{i 2}\right) \pi(\tau) \pi(\lambda) \pi(\rho) \\
& =\frac{\tau^{n-1}\left(1-\rho^{2}\right)^{n}}{(2 \pi)^{n}(1-a) \lambda} \exp \left[-\frac{\tau\left(1-\rho^{2}\right)}{2} \sum_{i=1}^{n}\left\{\left(\mu_{i 1}-y_{i 0}\right)^{2}+\left(\mu_{i 2}-y_{i 0}\right)^{2}\right\}\right]
\end{aligned}
$$

where $\Theta=\left\{\mu_{i 1}, \mu_{i 2}, \tau, \lambda, \rho\right\}$. The posterior probability under each hypothesis can be define by its likelihood function and prior information of the parameters under consideration.

\section{Posterior Probability}

Main motive behind the present study is to test the unit root hypothesis in consideration of break in mean and variance or both. For testing the hypothesis, we have derived posterior odds ratio using posterior probabilities for all possible models under various hypothesis which are listed in section (2) under the equations from (2.3) to (2.8). The posterior probabilities are presented by theorems 4.1 to 4.6 , for which we use the 
following notations are given below:

$$
\begin{aligned}
& M(\rho)=T_{B}(1-\rho)^{2}+\left(1-\rho^{2}\right) \\
& K(\rho)=(1-\rho) \sum_{t=1}^{T_{B}}\left(y_{i t}-\rho y_{i, t-1}\right)+\left(1-\rho^{2}\right) y_{i 0} \\
& G(\rho, \lambda)=\left(\frac{T-T_{B}}{\lambda}\right)(1-\rho)^{2}+\left(1-\rho^{2}\right) \\
& H(\rho, \lambda)=\left(\frac{1-\rho}{\lambda}\right) \sum_{t=T_{B}+1}^{T}\left(y_{i t}-\rho y_{i, t-1}\right)+\left(1-\rho^{2}\right) y_{i 0} \\
& I(\rho, \lambda)=\sum_{i=1}^{n} \sum_{t=1}^{T_{B}}\left(y_{i t}-\rho y_{i, t-1}\right)^{2}+\frac{1}{\lambda} \sum_{i=1}^{n} \sum_{t=T_{B}+1}^{T}\left(y_{i t}-\rho y_{i, t-1}\right)^{2} \\
& +2\left(1-\rho^{2}\right) \sum_{i=1}^{n} y_{i 0}^{2}-\sum_{i=1}^{n} \frac{[K(\rho)]^{2}}{M(\rho)}-\sum_{i=1}^{n} \frac{[H(\rho, \lambda)]^{2}}{G(\rho, \lambda)} \\
& S(\lambda)=\sum_{i=1}^{n} \sum_{t=1}^{T_{B}}\left(y_{i t}-y_{i, t-1}\right)^{2}+\frac{1}{\lambda} \sum_{i=1}^{n} \sum_{t=T_{B}+1}^{T}\left(y_{i t}-y_{i, t-1}\right)^{2} \\
& A(\rho, \lambda)=T_{B}(1-\rho)^{2}+\left(\frac{T-T_{B}}{\lambda}\right)(1-\rho)^{2}+\left(1-\rho^{2}\right) \\
& B(\rho, \lambda)=(1-\rho) \sum_{t=1}^{T_{B}}\left(y_{i t}-\rho y_{i, t-1}\right)+\left(\frac{1-\rho}{\lambda}\right) \sum_{t=T_{B}+1}^{T}\left(y_{i t}-\rho y_{i, t-1}\right) \\
& +\left(1-\rho^{2}\right) y_{i 0} \\
& C(\rho, \lambda)=\sum_{i=1}^{n} \sum_{t=1}^{T_{B}}\left(y_{i t}-\rho y_{i, t-1}\right)^{2}+\frac{1}{\lambda} \sum_{i=1}^{n} \sum_{t=T_{B}+1}^{T}\left(y_{i t}-\rho y_{i, t-1}\right)^{2} \\
& +\left(1-\rho^{2}\right) \sum_{i=1}^{n} y_{i 0}^{2}-\sum_{i=1}^{n} \frac{[B(\rho, \lambda)]^{2}}{A(\rho, \lambda)} \\
& N(\rho)=\left(T-T_{B}\right)(1-\rho)^{2}+\left(1-\rho^{2}\right) \\
& L(\rho)=(1-\rho) \sum_{t=T_{B}+1}^{T}\left(y_{i t}-\rho y_{i, t-1}\right)+\left(1-\rho^{2}\right) y_{i 0} \\
& O(\rho)=\sum_{i=1}^{n}\left[\sum_{t=1}^{T}\left(y_{i t}-\rho y_{i, t-1}\right)^{2}+2\left(1-\rho^{2}\right) y_{i 0}^{2}-\frac{[K(\rho)]^{2}}{M(\rho)}-\frac{[L(\rho)]^{2}}{N(\rho)}\right] \\
& P(\rho)=T(1-\rho)^{2}+\left(1-\rho^{2}\right) \\
& Q(\rho)=(1-\rho) \sum_{t=1}^{T}\left(y_{i t}-\rho y_{i, t-1}\right)+\left(1-\rho^{2}\right) y_{i 0} \\
& R(\rho)=\sum_{i=1}^{n} \sum_{t=1}^{T}\left(y_{i t}-\rho y_{i, t-1}\right)^{2}+\left(1-\rho^{2}\right) \sum_{i=1}^{n} y_{i 0}^{2}-\sum_{i=1}^{n} \frac{[Q(\rho)]^{2}}{P(\rho)}
\end{aligned}
$$


4.1. Theorem. The posterior probability under $H^{1}$, model is stationary with break in mean and variance at same and known point of time $T_{B}$, is given by

$$
P\left(y \mid H^{1}\right)=\int_{a}^{1} \int_{0}^{\infty} \frac{\left(1-\rho^{2}\right)^{n} \Gamma\left(\frac{n T}{2}\right)}{(\pi)^{\frac{n T}{2}}(1-a) \lambda[M(\rho)]^{\frac{n}{2}}[G(\rho, \lambda)]^{\frac{n}{2}}[I(\rho, \lambda)]^{\frac{n T}{2}}} d \lambda d \rho
$$

Proof. Proof is given in Appendix A1.

4.2. Theorem. The posterior probability under $H^{2}$ or $H^{3}$, in which model is difference stationary with known break in mean and variance, is given by

$$
P\left(y \mid H^{2}\right)=P\left(y \mid H^{3}\right)=\int_{0}^{\infty} \frac{\Gamma\left(\frac{n T}{2}\right)}{(\pi)^{\frac{n T}{2}} \lambda[S(\lambda)]^{\frac{n T}{2}}} d \lambda
$$

Proof. Proof is given in Appendix A2.

4.3. Theorem. The posterior probability under $H^{4}$, when model is stationary with single known break in variance, is given by

$$
P\left(y \mid H^{4}\right)=\int_{a}^{1} \int_{0}^{\infty} \frac{\left(1-\rho^{2}\right)^{\frac{n}{2}} \Gamma\left(\frac{n T}{2}\right)}{(\pi)^{\frac{n T}{2}}(1-a) \lambda[A(\rho, \lambda)]^{\frac{n}{2}}[C(\rho, \lambda)]^{\frac{n T}{2}}} d \lambda d \rho
$$

Proof. Proof is given in Appendix A3.

4.4. Theorem. Under $H^{5}$ model is stationary in which we consider the break in mean only. The posterior probability is given by

$$
P\left(y \mid H^{5}\right)=\int_{a}^{1} \frac{\left(1-\rho^{2}\right)^{n} \Gamma\left(\frac{n T}{2}\right)}{(\pi)^{\frac{n T}{2}}(1-a)[M(\rho)]^{\frac{n}{2}}[N(\rho)]^{\frac{n}{2}}[O(\rho)]^{\frac{n T}{2}}} d \rho
$$

Proof. Proof is given in Appendix A4.

4.5. Theorem. Model is difference stationary equivalently hypothesis is $H^{6}$ or $H^{7}$. The posterior probability is given by

$$
P\left(y \mid H^{6}\right)=P\left(y \mid H^{7}\right)=\frac{\Gamma\left(\frac{n T}{2}\right)}{(\pi)^{\frac{n T}{2}}\left[\sum_{i=1}^{n} \sum_{t=1}^{T}\left(y_{i t}-y_{i, t-1}\right)^{2}\right]^{\frac{n T}{2}}}
$$

Proof. Proof is given in Appendix A5.

4.6. Theorem. Model is stationary with no structural break and we get the posterior probability is

$$
P\left(y \mid H^{8}\right)=\int_{a}^{1} \frac{\left(1-\rho^{2}\right)^{\frac{n}{2}} \Gamma\left(\frac{n T}{2}\right)}{(\pi)^{\frac{n T}{2}}(1-a)[P(\rho)]^{\frac{n}{2}}[R(\rho)]^{\frac{n T}{2}}} d \rho
$$

Proof. Proof is given in Appendix A6. 


\section{Posterior Odds Ratio}

In Bayesian testing procedure, we use the probability in favor of one statement i.e. null hypothesis against the other statement i.e. alternative hypothesis. The comparison of two different hypothesis under Bayesian inference can be made by using posterior odds ratio $\left(\beta_{01}\right)$ and it is given as (Kass and Raftery [8])

$$
\beta_{01}=\frac{P\left(H_{0} \mid y\right)}{P\left(H_{1} \mid y\right)}=O\left(H_{0}\right) \frac{P\left(y \mid H_{0}\right)}{P\left(y \mid H_{1}\right)}=\frac{\theta}{1-\theta} \frac{P\left(y \mid H_{0}\right)}{P\left(y \mid H_{1}\right)}
$$

For comparing models, posterior probabilities of all models can be used as a measure for testing the hypothesis. Then, one can obtain posterior odds ratio using these posterior probabilities, under various combinations of null and alternative hypothesis like series is stationary or difference stationary with break or no break. Using all these posterior probabilities, we may obtain the posterior odds ratio for all possible combination of null as well as alternative hypothesis is listed from $\operatorname{POR}_{1}\left(\beta_{01}^{1}\right)$ to $P O R_{14}\left(\beta_{01}^{14}\right)$.

$\boldsymbol{P O R}_{1}$ : For testing the null hypothesis $H^{2}: \rho=1, \mu_{i 1} \neq \mu_{i 2}, \lambda \neq 1$ or $H^{3}: \rho=$ $1, \mu_{i 1}=\mu_{i 2}, \lambda \neq 1$ i.e., series is difference stationary with break in variance against the alternative hypothesis $H^{1}: \rho \epsilon s, \mu_{i 1} \neq \mu_{i 2}, \lambda \neq 1$ i.e., series is stationary under the consideration of break in both mean and variance. Then, POR is given as

$$
\beta_{01}^{1}=\frac{\theta}{1-\theta} \frac{\int_{0}^{\infty} \frac{1}{\lambda[S(\lambda)]^{\frac{n T}{2}}} d \lambda}{\int_{a}^{1} \int_{0}^{\infty} \frac{\left(1-\rho^{2}\right)^{n}}{(1-a) \lambda[M(\rho)]^{\frac{n}{2}}[G(\rho, \lambda)]^{\frac{n}{2}}[I(\rho, \lambda)]^{\frac{n T}{2}}} d \lambda d \rho}
$$

$\boldsymbol{P O R}_{2}$ : For testing the null hypothesis $H^{4}: \rho \epsilon s, \mu_{i 1}=\mu_{i 2}, \lambda \neq 1$, series is trend stationary with break in variance against the alternative hypothesis $H^{1}: \rho \epsilon s, \mu_{i 1} \neq$ $\mu_{i 2}, \lambda \neq 1$, series is stationary when break occurs on both. Then, POR is given as

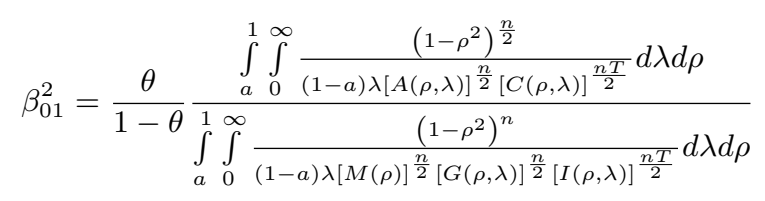

$\boldsymbol{P O R}_{3}$ : For testing the null hypothesis $H^{5}: \rho \epsilon s, \mu_{i 1} \neq \mu_{i 2}, \lambda=1$, series is trend stationary with break in mean against the alternative hypothesis $H^{1}: \rho \epsilon s, \mu_{i 1} \neq$ $\mu_{i 2}, \lambda \neq 1$, series is stationary under consideration of break occurs in mean as well as variance. Then, POR is given as

$$
\beta_{01}^{3}=\frac{\theta}{1-\theta} \frac{\int_{a}^{1} \frac{\left(1-\rho^{2}\right)^{n}}{(1-a)[M(\rho)]^{\frac{n}{2}}[N(\rho)]^{\frac{n}{2}}[O(\rho)]^{\frac{n T}{2}}} d \rho}{\left(1-\rho^{2}\right)^{n}} \int_{0}^{\infty} \frac{(1-a) \lambda[M(\rho)]^{\frac{n}{2}}[G(\rho, \lambda)]^{\frac{n}{2}}[I(\rho, \lambda)]^{\frac{n T}{2}}}{(\lambda) d \rho}
$$

$\boldsymbol{P O R}_{4}$ : For testing the null hypothesis $H^{6}: \rho=1, \mu_{i 1} \neq \mu_{i 2}, \lambda=1$ or $H^{7}: \rho=1, \mu_{i 1}=$ $\mu_{i 2}, \lambda=1$ series is difference stationary with no break against the alternative hypothesis $H^{1}: \rho \epsilon s, \mu_{i 1} \neq \mu_{i 2}, \lambda \neq 1$, series is stationary when break in both. Then, POR is given as

$$
\beta_{01}^{4}=\frac{\theta}{1-\theta} \frac{\left[\sum_{i=1}^{n} \sum_{t=1}^{T}\left(y_{i t}-y_{i, t-1}\right)^{2}\right]^{-\frac{n T}{2}}}{\int_{a}^{1} \int_{0}^{\infty} \frac{\left(1-\rho^{2}\right)^{n}}{(1-a) \lambda[M(\rho)]^{\frac{n}{2}}[G(\rho, \lambda)]^{\frac{n}{2}}[I(\rho, \lambda)]^{\frac{n T}{2}}} d \lambda d \rho}
$$


$\boldsymbol{P O R}_{5}$ : For testing the null hypothesis $H^{8}: \rho \epsilon s, \mu_{i 1}=\mu_{i 2}, \lambda=1$, series is trend stationary with no break against the alternative hypothesis $H^{1}: \rho \epsilon s, \mu_{i 1} \neq \mu_{i 2}, \lambda \neq 1$, series is stationary under consideration of break is present in both. Then, POR is given as

$$
\beta_{01}^{5}=\frac{\theta}{1-\theta} \frac{\int_{a}^{1} \frac{\left(1-\rho^{2}\right)^{\frac{n}{2}}}{(1-a)[P(\rho)]^{\frac{n}{2}}[R(\rho)]^{\frac{n T}{2}}} d \rho}{\left(1-\rho^{2}\right)^{n}} \int_{0}^{\infty} \frac{]_{0}^{\frac{n}{2}}[I(\rho, \lambda)]^{\frac{n T}{2}}}{(1-a) \lambda[M(\rho)]^{\frac{n}{2}}[G(\rho, \lambda)]^{\frac{n}{2}} d \rho}
$$

$\boldsymbol{P O R}_{6}$ : For testing the null hypothesis $H^{2}: \rho=1, \mu_{i 1} \neq \mu_{i 2}, \lambda \neq 1$ or $H^{3}: \rho=1, \mu_{i 1}=$ $\mu_{i 2}, \lambda \neq 1$ series is difference stationary with break in variance against the alternative hypothesis $H^{4}: \rho \epsilon s, \mu_{i 1}=\mu_{i 2}, \lambda \neq 1$, series is stationary under consideration of break in variance only. Then, POR is given as

$$
\beta_{01}^{6}=\frac{\theta}{1-\theta} \frac{\int_{0}^{\infty} \frac{1}{\lambda[S(\lambda)]^{\frac{n T}{2}}} d \lambda}{\int_{a}^{1} \int_{0}^{\infty} \frac{\left(1-\rho^{2}\right)^{\frac{n}{2}}}{(1-a) \lambda[A(\rho, \lambda)]^{\frac{n}{2}}[C(\rho, \lambda)]^{\frac{n T}{2}}} d \lambda d \rho}
$$

$\boldsymbol{P O R}_{7}$ : For testing the null hypothesis $H^{5}: \rho \epsilon s, \mu_{i 1} \neq \mu_{i 2}, \lambda=1$, series is trend stationary with break in mean against the alternative hypothesis $H^{4}: \rho \epsilon s, \mu_{i 1}=$ $\mu_{i 2}, \lambda \neq 1$, series is stationary under break in variance. Then, POR is given as

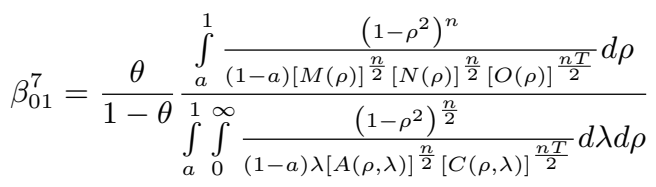

$\boldsymbol{P O R}_{8}$ : For testing the null hypothesis $H^{6}: \rho=1, \mu_{i 1} \neq \mu_{i 2}, \lambda=1$ or $H^{7}: \rho=1, \mu_{i 1}=$ $\mu_{i 2}, \lambda=1$ series is difference stationary with no break against the alternative hypothesis $H^{4}: \rho \epsilon s, \mu_{i 1}=\mu_{i 2}, \lambda \neq 1$, series is stationary under consideration of break in variance. Then, POR is given as

$$
\beta_{01}^{8}=\frac{\theta}{1-\theta} \frac{\left[\sum_{i=1}^{n} \sum_{t=1}^{T}\left(y_{i t}-y_{i, t-1}\right)^{2}\right]^{-\frac{n T}{2}}}{\int_{a}^{1} \int_{0}^{\infty} \frac{\left(1-\rho^{2}\right)^{\frac{n}{2}}}{(1-a) \lambda[A(\rho, \lambda)]^{\frac{n}{2}}[C(\rho, \lambda)]^{\frac{n T}{2}}} d \lambda d \rho}
$$

$\boldsymbol{P O R}_{9}$ : For testing the null hypothesis $H^{8}: \rho \epsilon s, \mu_{i 1}=\mu_{i 2}, \lambda=1$, series is trend stationary with no break against the alternative hypothesis $H^{4}: \rho \epsilon s, \mu_{i 1}=\mu_{i 2}, \lambda \neq 1$, series is stationary under the consideration of break in variance. Then, POR is given as

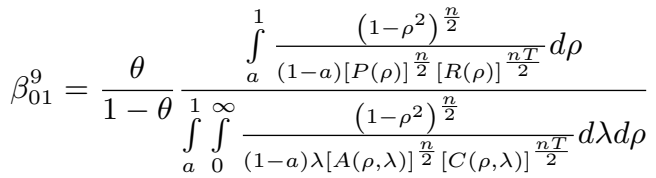

$\boldsymbol{P O R}_{10}$ : For testing the null hypothesis $H^{6}: \rho=1, \mu_{i 1} \neq \mu_{i 2}, \lambda=1$ or $H^{7}: \rho=1, \mu_{i 1}=$ $\mu_{i 2}, \lambda=1$, series is difference stationary with no break against the alternative hypothesis $H^{5}: \rho \epsilon s, \mu_{i 1} \neq \mu_{i 2}, \lambda=1$, series is stationary under break in mean. Then, POR is 
given as

$$
\beta_{01}^{10}=\frac{\theta}{1-\theta} \frac{\left[\sum_{i=1}^{n} \sum_{t=1}^{T}\left(y_{i t}-y_{i, t-1}\right)^{2}\right]^{-\frac{n T}{2}}}{\int_{a}^{1} \frac{\left(1-\rho^{2}\right)^{n}}{(1-a)[M(\rho)]^{\frac{n}{2}}[N(\rho)]^{\frac{n}{2}}[O(\rho)]^{\frac{n T}{2}}} d \rho}
$$

$\boldsymbol{P O R}_{11}$ : For testing the null hypothesis $H^{8}: \rho \epsilon s, \mu_{i 1}=\mu_{i 2}, \lambda=1$, series is stationary with no break against the alternative hypothesis $H^{5}: \rho \epsilon s, \mu_{i 1} \neq \mu_{i 2}, \lambda=1$, series is stationary under break in mean. Then, POR is given as

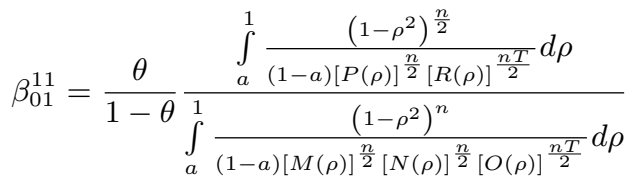

$\boldsymbol{P O R}_{12}$ : For testing the null hypothesis $H^{2}: \rho=1, \mu_{i 1} \neq \mu_{i 2}, \lambda \neq 1$ or $H^{3}: \rho=1, \mu_{i 1}=$ $\mu_{i 2}, \lambda \neq 1$, series is difference stationary with break in variance against the alternative hypothesis $H^{5}: \rho \epsilon s, \mu_{i 1} \neq \mu_{i 2}, \lambda=1$, series is stationary under break in mean. Then, $\mathrm{POR}$ is given as

$$
\beta_{01}^{12}=\frac{\theta}{1-\theta} \frac{\int_{0}^{\infty} \frac{1}{\lambda[S(\lambda)]^{\frac{n T}{2}}} d \lambda}{\int_{a}^{1} \frac{\left(1-\rho^{2}\right)^{n}}{(1-a)[M(\rho)]^{\frac{n}{2}}[N(\rho)]^{\frac{n}{2}}[O(\rho)]^{\frac{n T}{2}}} d \rho}
$$

$\boldsymbol{P O R}_{13}$ : For testing the null hypothesis $H^{2}: \rho=1, \mu_{i 1} \neq \mu_{i 2}, \lambda \neq 1$ or $H^{3}: \rho=1, \mu_{i 1}=$ $\mu_{i 2}, \lambda \neq 1$, series is difference stationary with break in variance against the alternative hypothesis $H^{8}: \rho \epsilon s, \mu_{i 1}=\mu_{i 2}, \lambda=1$, series is stationary under no break. Then, POR is given as

$$
\beta_{01}^{13}=\frac{\theta}{1-\theta} \frac{\int_{0}^{\infty} \frac{1}{\lambda[S(\lambda)]^{\frac{n T}{2}}} d \lambda}{\int_{a}^{1} \frac{\left(1-\rho^{2}\right)^{\frac{n}{2}}}{(1-a)[P(\rho)]^{\frac{n}{2}}[R(\rho)]^{\frac{n T}{2}}} d \rho}
$$

$\boldsymbol{P O R}_{14}$ : For testing the null hypothesis $H^{6}: \rho=1, \mu_{i 1} \neq \mu_{i 2}, \lambda=1$ or $H^{7}: \rho=1, \mu_{i 1}=$ $\mu_{i 2}, \lambda=1$, series is difference stationary with no break against the alternative hypothesis $H^{8}: \rho \epsilon s, \mu_{i 1}=\mu_{i 2}, \lambda=1$, series is stationary under no break. Then, POR is given as

$$
\beta_{01}^{14}=\frac{\theta}{1-\theta} \frac{\left[\sum_{i=1}^{n} \sum_{t=1}^{T}\left(y_{i t}-y_{i, t-1}\right)^{2}\right]^{-\frac{n T}{2}}}{\int_{a}^{1} \frac{\left(1-\rho^{2}\right)^{\frac{n}{2}}}{(1-a)[P(\rho)]^{\frac{n}{2}}[R(\rho)]^{\frac{n T}{2}}} d \rho}
$$

\section{Numerical Illustrations}

Structural break in the model occurs because of various reasons like change on policy, management or any other social or political changes and any time series may be affected by them. In present study our main purpose is to studying $\operatorname{PAR}(1)$ time series model considering both type of breaks i.e. break in mean and variance given by (2.3). For understanding the impact of the break, we have performed simulation and empirical analysis. This may be misinterpreted by various forms given by (2.4) to (2.8) and one may also be interested to study the behavior in respect to a particular form for various hypothesis. 

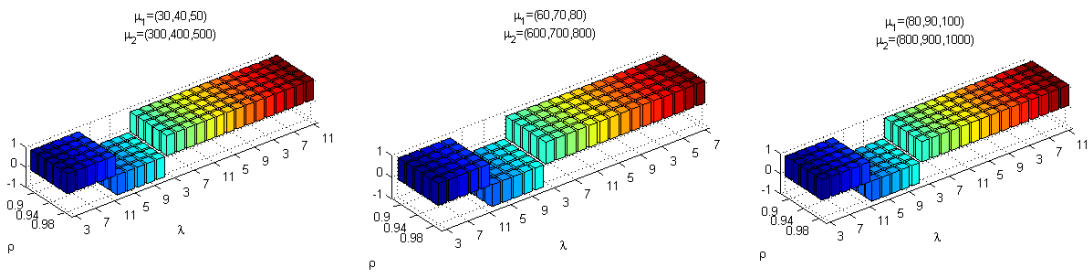

Figure 1. When alternative hypothesis considers break on mean and variance both

6.1. Simulation Study. A simulation study is carried out in which we have generated panel data time series model of size $\mathrm{T}=25$ considering three panels with the initial observations $\left[y_{10}, y_{20}, y_{30}\right]=[100,120,140]$ and taken break point in each panel at $T_{B}=15$. Our main interest is to explore PAR(1) in respect to unit root hypothesis in association with break in mean and variance. Therefore we have explored our study for various autoregressive models using coefficient $\rho=[0.90,0.92,0.95,0.98,0.99], \lambda=[3,5,7,9$, 11] with error variance $\tau=0.025, \mu_{i 1}$ is the mean before the break considering three combinations $[(30,40,50) ;(60,70,80) ;(80,90,100)]$ and $\mu_{i 2}$ is the mean after the break considering three combinations [(300,400,500); $(600,700,800) ;(800,900,1000)]$. For the posterior probability, expression need's to integrate in respect to $\rho$ and $\lambda$. For this, we used Monte Carlo integration method taking 5000 values of $\rho$ from $\mathrm{U}(\mathrm{a}, 1)$ and $\lambda$ from $\mathrm{U}(0,1)$, where a is obtained by Dickey Fuller test statistic.

Model (2.3) can be written by a particular model (i) break on both, (ii) break only in variance (iii) break only in mean and (iv) no break in consideration of trend stationary as well as difference stationary series. All cases from (i) to (iv) are tested, taking various null hypothesis which are considered with the posterior odds ratio listed by $P O R_{1}$ to $P O R_{14}$. As there are several values and our purpose is to test the null against the alternative and conclude the inference therein. The POR value which we get is too small to identify the accept-reject criterion and do not give better interpretation. For making appropriate conclusion, we interpreted the result by using graphically. In the figures, $\mathrm{x}$-axis and $\mathrm{y}$-axis denote the different values of $\lambda, \rho$ and $\mathrm{z}$-axis considering the decision rule to accept or reject the null hypothesis. If the value of POR is less than one means reject the null hypothesis, denoted by 1 otherwise accepted, denoted by -1 . In details all cases are discussed below:

\section{Case 1: Both break on mean and variance}

The true model with generated model is considering break in both mean and variance. First we have taken it as alternative and testing for all possible null by using theorem 4.1 to 4.6 which is given in section (4) .We have tested the null hypothesis against similar alternative hypothesis one by one and obtained the POR from $P O R_{1}$ to $P O R_{5}$. The results obtained for simulated data are very small and cannot be represented due to of size limitation, therefore we are presenting here graphically. In the Figure 1, considering all hypotheses under the given model in equations from (2.4) to (2.8) as a null and the alternative hypothesis are set by equation (2.3) i.e. hypothesis associated with true model which allows structural break in both mean and variance. We observed that except $H^{4}$, other null hypothesis under the true model is rejected which advocate that both break provide more impact on series to decrease the POR. In hypothesis $H^{4}$, we ignore break in the intercept which increase fluctuation and then impact the value of the error variance. 

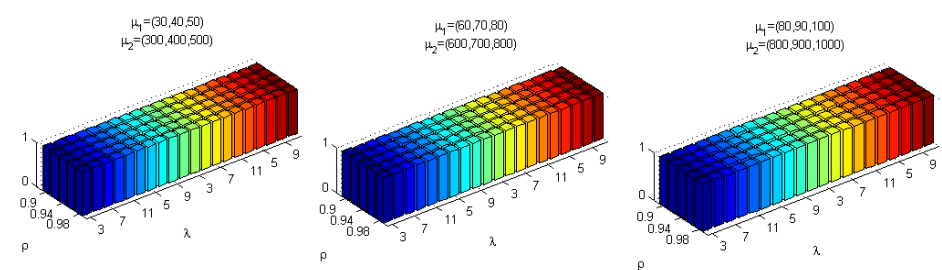

Figure 2. When alternative hypothesis considers break on variance

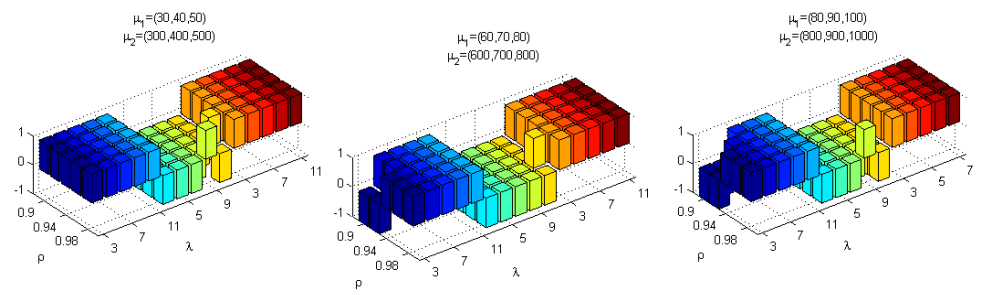

Figure 3. When alternative hypothesis considers break on mean

This reduces the value of POR and concludes that alternative hypothesis is rejected. It is easily observed from the figure, rejection criterion does not much affected by large value of $\rho$ and $\lambda$ as well as different values of intercept term do not affect the acceptance or rejection criterion.

\section{Case 2: Break in variance}

If we ignore break in mean, hypothesis under the true model is coming only due to break in error variance and testing with other null hypothesis be carried out. We know that error term affect the series more than other terms like linear trend, intercept etc. If break is also present in the error term, then fluctuation of the series after the break point is more and this directly impact the model. Therefore, the POR is always less than 1 . In Figure 2, here it is observed that all null hypotheses are rejected whatever the series is stationary or non-stationary with break in mean or variance. Increase on value of intercept term and different values of $\lambda$ as well as $\rho$ do not change our rejection or acceptance of the hypothesis. Similarly, ignoring break in mean is also not affecting the unit root hypothesis.

\section{Case 3: Break in mean}

Present case considers the break in mean only i.e. we ignore the break in variance. We have presented the result in Figure 3. Here it is revealed the proposed hypothesis significantly affecting the correct model for large value of $\rho$ and $\lambda$ i.e. alternative hypothesis is accepted. But if series is stationary with no break comparison to our assumed hypothesis, the POR value is greater than one and null is accepted. Under unit root hypothesis with break in mean does not affect much because mean term is vanishes from the model. Here, ignoring break in error variance of models may increase the strength of evidence for a unit root against stationary when an alternative model has break in mean term. This is also justified from the present simulation study. 

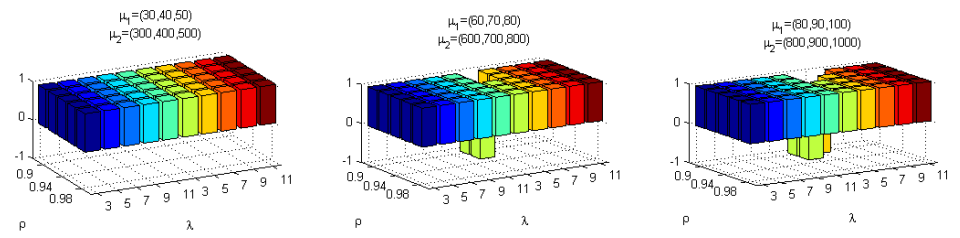

Figure 4. When alternative hypothesis considers no break

\section{Case 4: No break is consider}

There are several examples where break is not taken into account. Considering this argument we have tested the model without any break and results are presented by Figure 4. This figure present decision between the unit root against stationary hypothesis. The first hypothesis $H^{2}$ or $H^{3}$ is rejected for every value of $\mu, \rho$ and $\lambda$ whereas the second hypothesis $H^{6}$ or $H^{7}$ is accepted for small value of $\rho$ and $\lambda$ with increasing value of $\mu$. It is clear from the Figure 4, unit root hypothesis is rejected for all the case because break in both is ignored and there is coefficient on upper direction which will defiantly increase the autoregressive coefficients value. It is also observed that if series is stationary under break or no break provides less POR value as compare to unit root hypothesis.

6.2. Empirical Study. In present section, we have done empirical analysis of our proposed model to get better interpretation and target then to get justify the proposed study. For a nation, agriculture product is one of the necessary elements to observe the economic growth and mainly changeable according to the season. But it also depends upon average land use, quality of seeds, better use of fertilizers, new technology etc and it affects the production of the outputs. Therefore, we proposed a panel data time series model which is handling the change in the production of agriculture commodities when series having structural break. For which we have taken data from Agricultural Statistics at a Glance 2014, Source data from Department of Fertilizers, Ministry of Chemicals \& Fertilizers. In this data set, we analyzed the import of fertilizers, name as N (Nitrogen), P (Phosphate), K (Potash) cover the period from 1980-81 to 2013-14. First of all, we identified the structural break in the import series using $\mathrm{R}$ software. Command strucchange recognizes break point for individual series is developed by (Zeileis et al. [24]) and recorded the break point of individual series reported in the Table 1 are given below.

Table 1. Structural break point present in different fertilizers

\begin{tabular}{cccc}
\hline Break point & \multicolumn{3}{c}{ Fertilizers } \\
\cline { 2 - 4 } & $\mathbf{N}$ & $\mathbf{P}$ & $\mathbf{K}$ \\
\hline $\mathrm{T} 1$ & 24 & 25 & 16 \\
$\mathrm{~T} 2$ & 28 & - & 24 \\
\hline
\end{tabular}

Here it is observed that the range of break point is between 16 to 28 . Therefore, we have tested our model considering every time point as a break point in this interval. The model has explored first fitting for the import data of fertilizers and tested the unit root hypothesis under all fours cases using the derived POR listed by $P O R_{1}$ to $P O R_{14}$.

Table 2 shows that if break is present in mean and variance both, the posterior odds ratio value is too small to reject the null hypothesis except null hypothesis is considers only break in variance. This may be happened due to direct impact of the error variance 
Table 2. Posterior odds ratio value when alternative hypothesis consider break in both

\begin{tabular}{cccccc}
\hline Break Point & $\beta_{01}^{1}$ & $\beta_{01}^{2}$ & $\beta_{01}^{3}$ & $\beta_{01}^{4}$ & $\beta_{01}^{5}$ \\
\hline 16 & $7.24 \mathrm{E}-31$ & 1.4100 & $3.09 \mathrm{E}-30$ & $2.13 \mathrm{E}-29$ & $3.73 \mathrm{E}-30$ \\
17 & $7.11 \mathrm{E}-29$ & 2.5025 & $4.32 \mathrm{E}-28$ & $2.81 \mathrm{E}-27$ & $5.16 \mathrm{E}-28$ \\
18 & $2.12 \mathrm{E}-27$ & 2.6595 & $1.11 \mathrm{E}-26$ & $8.48 \mathrm{E}-26$ & $1.39 \mathrm{E}-26$ \\
19 & $1.98 \mathrm{E}-25$ & 3.3339 & $9.11 \mathrm{E}-25$ & $5.83 \mathrm{E}-24$ & $1.12 \mathrm{E}-24$ \\
20 & $8.11 \mathrm{E}-25$ & 2.5833 & $4.25 \mathrm{E}-24$ & $2.85 \mathrm{E}-23$ & $5.14 \mathrm{E}-24$ \\
21 & $9.58 \mathrm{E}-25$ & 1.9031 & $4.99 \mathrm{E}-24$ & $3.02 \mathrm{E}-23$ & $5.99 \mathrm{E}-24$ \\
22 & $1.64 \mathrm{E}-24$ & 1.5227 & $6.46 \mathrm{E}-24$ & $5.14 \mathrm{E}-23$ & $7.85 \mathrm{E}-24$ \\
23 & $2.17 \mathrm{E}-24$ & 3.0106 & $1.04 \mathrm{E}-23$ & $6.70 \mathrm{E}-23$ & $1.23 \mathrm{E}-23$ \\
24 & $1.98 \mathrm{E}-20$ & 1.7244 & $9.23 \mathrm{E}-20$ & $6.15 \mathrm{E}-19$ & $1.03 \mathrm{E}-19$ \\
25 & $4.11 \mathrm{E}-17$ & 1.1200 & $1.54 \mathrm{E}-16$ & $1.04 \mathrm{E}-15$ & $1.79 \mathrm{E}-16$ \\
26 & $1.99 \mathrm{E}-14$ & 1.2738 & $6.99 \mathrm{E}-14$ & $5.20 \mathrm{E}-13$ & $7.68 \mathrm{E}-14$ \\
27 & $2.47 \mathrm{E}-10$ & 1.2153 & $8.57 \mathrm{E}-10$ & $4.90 \mathrm{E}-09$ & $8.31 \mathrm{E}-10$ \\
28 & $1.10 \mathrm{E}-10$ & 1.1944 & $3.67 \mathrm{E}-10$ & $2.07 \mathrm{E}-09$ & $3.72 \mathrm{E}-10$ \\
\hline
\end{tabular}

in the series and it can also be analyzed by ignoring the mean term. Therefore, the import of fertilizers series is stationary either break in mean and variance or break in variance only.

Table 3. Posterior odds ratio value when alternative hypothesis consider break in variance

\begin{tabular}{ccccc}
\hline Break Point & $\beta_{01}^{6}$ & $\beta_{01}^{7}$ & $\beta_{01}^{8}$ & $\beta_{01}^{9}$ \\
\hline 16 & $5.13 \mathrm{E}-31$ & $2.19 \mathrm{E}-30$ & $1.51 \mathrm{E}-29$ & $2.65 \mathrm{E}-30$ \\
17 & $2.84 \mathrm{E}-29$ & $1.73 \mathrm{E}-28$ & $1.12 \mathrm{E}-27$ & $2.06 \mathrm{E}-28$ \\
18 & $7.96 \mathrm{E}-28$ & $4.18 \mathrm{E}-27$ & $3.19 \mathrm{E}-26$ & $5.22 \mathrm{E}-27$ \\
19 & $5.95 \mathrm{E}-26$ & $2.73 \mathrm{E}-25$ & $1.75 \mathrm{E}-24$ & $3.37 \mathrm{E}-25$ \\
20 & $3.14 \mathrm{E}-25$ & $1.64 \mathrm{E}-24$ & $1.10 \mathrm{E}-23$ & $1.99 \mathrm{E}-24$ \\
21 & $5.04 \mathrm{E}-25$ & $2.62 \mathrm{E}-24$ & $1.59 \mathrm{E}-23$ & $3.15 \mathrm{E}-24$ \\
22 & $1.08 \mathrm{E}-24$ & $4.24 \mathrm{E}-24$ & $3.38 \mathrm{E}-23$ & $5.15 \mathrm{E}-24$ \\
23 & $7.22 \mathrm{E}-25$ & $3.47 \mathrm{E}-24$ & $2.23 \mathrm{E}-23$ & $4.10 \mathrm{E}-24$ \\
24 & $1.15 \mathrm{E}-20$ & $5.35 \mathrm{E}-20$ & $3.57 \mathrm{E}-19$ & $5.97 \mathrm{E}-20$ \\
25 & $3.67 \mathrm{E}-17$ & $1.37 \mathrm{E}-16$ & $9.29 \mathrm{E}-16$ & $1.60 \mathrm{E}-16$ \\
26 & $1.56 \mathrm{E}-14$ & $5.49 \mathrm{E}-14$ & $4.08 \mathrm{E}-13$ & $6.03 \mathrm{E}-14$ \\
27 & $2.03 \mathrm{E}-10$ & $7.05 \mathrm{E}-10$ & $4.03 \mathrm{E}-09$ & $6.84 \mathrm{E}-10$ \\
28 & $9.20 \mathrm{E}-11$ & $3.07 \mathrm{E}-10$ & $1.73 \mathrm{E}-09$ & $3.11 \mathrm{E}-10$ \\
\hline
\end{tabular}

In Table 3, we consider the alternative hypothesis which has break only in variance. The POR is too small definitely less than 1 , so we reject the null hypothesis. This may be due to break in variance in error term and fluctuating the series to decrease the value of POR. It is concluded that import series is always stationary when break present only in variance.

Ignoring break in variance, the structure of the series may be changed by mean term which is reported in Table 4. In our first two cases, null hypothesis considers no break which can be concluded that series may be stationary or non-stationary but the third posterior odds ratio gets the series is difference stationary. Under unit root hypothesis with no break, series is non-stationary. 
Table 4. Posterior odds ratio value when alternative hypothesis consider break in mean

\begin{tabular}{cccc}
\hline Break Point & $\beta_{01}^{10}$ & $\beta_{01}^{11}$ & $\beta_{01}^{12}$ \\
\hline 16 & 6.8921 & 1.2078 & 0.2343 \\
17 & 6.5074 & 1.1961 & 0.1648 \\
18 & 7.6307 & 1.2495 & 0.1906 \\
19 & 6.4032 & 1.2327 & 0.2176 \\
20 & 6.6993 & 1.2099 & 0.1910 \\
21 & 6.0521 & 1.2008 & 0.1920 \\
22 & 7.9629 & 1.2147 & 0.2540 \\
23 & 6.4165 & 1.1821 & 0.2083 \\
24 & 6.6640 & 1.1148 & 0.2143 \\
25 & 6.7702 & 1.1659 & 0.2675 \\
26 & 7.4360 & 1.0992 & 0.2848 \\
27 & 5.7186 & 0.9697 & 0.2877 \\
28 & 5.6385 & 1.0126 & 0.2995 \\
\hline
\end{tabular}

Table 5. Posterior odds ratio value when alternative hypothesis consider no break

\begin{tabular}{ccc}
\hline Break Point & $\beta_{01}^{13}$ & $\beta_{01}^{14}$ \\
\hline 16 & 0.1940 & 5.7064 \\
17 & 0.1377 & 5.4405 \\
18 & 0.1525 & 6.1068 \\
19 & 0.1765 & 5.1945 \\
20 & 0.1579 & 5.5371 \\
21 & 0.1599 & 5.0401 \\
22 & 0.2091 & 6.5557 \\
23 & 0.1762 & 5.4281 \\
24 & 0.1922 & 5.9778 \\
25 & 0.2295 & 5.8068 \\
26 & 0.2591 & 6.7652 \\
27 & 0.2967 & 5.8973 \\
28 & 0.2957 & 5.5681 \\
\hline
\end{tabular}

In Table 5, no break is present in the series and get that series is non-stationary or unit root. The value of posterior odds ratio is less than one when break is present under unit root hypothesis and concluded that series is stationary. If no break is presence under unit root hypothesis to accept the null hypothesis, then series is concluded non-stationary.

The present empirical analysis is verifying the impact of structural break and reveals that if it is ignored then unit root hypothesis may be reversed. It is also observed that ignorance of break in variance is more prone to acceptance of unit root hypothesis. Here it is noted that break in mean is vanishes under the unit root case so this does not impact the model. It is also noticed that when both break are taken into account there are an increase on the value of POR. This can happen because of break, which is present in variance only under the unit root. The present study is strongly advocating that if break is not taken into account then unit root hypothesis get reverted. The similar result is also observed in simulated data. 


\section{Conclusion}

The present paper dealt the Bayesian analysis of PAR(1) time series model with break in mean and variance both. This is showing the importance of the analysis of structure break and recorded that the ignorance of break has serious impact on unit root hypothesis. The study may be extended for the model with non-normal error as well as model with non-linear time trend and then for multivariate time series model.

\section{Acknowledgement}

First author gratefully acknowledge the financial assistance from University Grant Commission, India under MRP Scheme(Grant No.42-43/2013).

\section{References}

[1] J. Bai. Common breaks in means and variances for panel data, Journal of Econometrics 157, 78-92, 2010

[2] B. H. Baltagi, Q. Feng and C. Kao. Estimation of heterogeneous panels with structural breaks, Journal of Econometrics 191(1), 176-195, 2016.

[3] G. E. P. Box and G. M. Jenkins. Time Series Analysis Forecasting and Control, Holden-Day: San Francisco, CA, 1970.

[4] A. Chaturvedi and J. Kumar. Bayesian unit root test for time series models with structural break, American Journal of Mathematical and Management Science 27(1), 243-268, 2007.

[5] S. Chib. Estimation and comparison of multiple changepoint models, Journal of Econometrics 86, 221-241, 1998 .

[6] S. Cook. Unit root testing in the presence of innovation variance breaks: a simple solution with increased power, Journal of Applied Mathematics 2(5), 233-240, 2002.

[7] C. Inclan. Detection of multiple changes of variance using posterior odds, Journal of Business \& Economic Statistics 11(3), 289-300, 1993.

[8] R. Kass and A. Raftery. Bayes factors, Journal of the American Statistical Association 90, 773-795, 1995.

[9] T. H. Kim, S. J. Leybourne and P. Newbold. Unit root test with a break in innovation variance, Journals of Econometrics 109, 365-387, 2002.

[10] T. Kim, B. Kim, H. Moon and S. Jeong. Unit root tests in the presence of multiple breaks in variance, The Singapore Economic Review 62(2), 345-361, 2017.

[11] R. Kumar, J. Kumar and A. Chaturvedi. Bayesian unit root test for time series models with structural break in variance, Journal of Economics and Econometrics 55(1), 75-86, 2012.

[12] A. Levin, C. F. Lin and C. S. J. Chu. Unit root test in panel data: asymptotic and finite sample properties, Journal of Econometrics 108(1), 1-24, 2002.

[13] D. Li, J. Qian and L. Su. Panel data models with interactive fixed effects and multiple structural breaks, Journal of the American Statistical Association 111(516), 1804-1819, 2016.

[14] L. Meligkotsidou, E. Tzavalis and I. D. Vrontos. A Bayesian analysis of unit roots and structural breaks in the level and the error variance of autoregressive models, Econometric Review 30(2), 208-249, 2011.

[15] P. Perron. Testing for a unit root in a time series with a changing mean, Journal of Business and Economic Statistics 8, 153-162, 1990.

[16] P. Perron. Further evidence on breaking trend functions in macroeconomic variables, Journal of Econometrics 80, 355-385, 1997.

[17] PP. Perron and T. J. Vogelsang. Nonstationarity and level shifts with an application to purchasing power parity, Journal of Business and Economic Statistics 10, 301-320, 1992.

[18] J. Qian, and L. Su. Shrinkage estimation of common breaks in panel data models via adaptive group fused lasso, Journal of Econometrics 191(1), 86-109, 2016.

[19] P. C. Schotman and H. K. Van Dijk. A Bayesian routes to unit roots, Journal of Applied Econometrics 6, 387-401, 1991.

[20] X. Shao and X. Zhang. Testing for change points in time series, Journal of the American Statistical Association 105(491), 1228-1240, 2010. 
[21] T. Vogelsang and P. Perron. Additional tests for a unit root allowing for a break in the trend function at an unknown time, International Economic Review 39(4), 1073-1100, 1998.

[22] J. Wang and E. Zivot. A Bayesian time series model of multiple structural changes in level, trend and variance, Journal of Business and Economic Statistics 18, 374-386, 2000.

[23] Y. Yao. Estimating the number of changepoints via Schwarz criterion, Statistics \& Probability Letters 6, 181-189, 1988.

[24] F. Zeileis, K. H. Leisch and C. Kleiber. Strucchange: an R package for testing for structural change in linear regression models, Journal of Statistical Software 7(2), 1-38, 2002.

[25] E. Zivot and D. W. K. Andrews. Further evidence on the great crash, the oil price shock, and the unit root hypothesis, Journal of Business and Economic Statistics 10, 251-270, 1992.

\section{Appendix}

Posterior probability under various setup of PAR(1) time series model consider break in mean and variance which are placed in equation (2.3) to (2.8). The likelihood function connected with the assumed prior distribution, the derived posterior probability under different model is given below.

A1: Posterior probability under $H^{1}$

In this hypothesis, the likelihood function containing break in mean as well as variance and is given by

$$
\begin{aligned}
L\left(\tau, \rho, \mu_{1}, \mu_{2}, \lambda \mid y\right)= & \frac{\tau^{\frac{n T}{2}}}{(2 \pi)^{\frac{n T}{2}}} \exp \left[-\frac{\tau}{2} \sum_{i=1}^{n}\left\{\sum_{t=1}^{T_{B}}\left(y_{i t}-\rho y_{i t-1}-(1-\rho) \mu_{i 1}\right)^{2}\right.\right. \\
& \left.\left.+\frac{1}{\lambda} \sum_{t=T_{B}+1}^{T}\left(y_{i t}-\rho y_{i t-1}-(1-\rho) \mu_{i 2}\right)^{2}\right\}\right]
\end{aligned}
$$

Combining the likelihood function with the prior distribution, the posterior probability

$$
\begin{aligned}
P\left(y \mid H^{1}\right)= & \int_{a}^{1} \int_{0}^{\infty} \int_{0}^{\infty} \int_{-\infty}^{\infty} \int_{-\infty}^{\infty} \frac{\tau^{\frac{n T}{2}+n-1}\left(1-\rho^{2}\right)^{n}}{(2 \pi)^{\frac{n T}{2}+n}(1-a) \lambda} \exp \left[-\frac{\tau}{2} \sum_{i=1}^{n}\left\{\sum_{t=1}^{T_{B}}\left(y_{i t}-\rho y_{i t-1}-(1-\rho) \mu_{i 1}\right)^{2}\right.\right. \\
& +\frac{1}{\lambda} \sum_{t=T_{B}+1}^{T}\left(y_{i t}-\rho y_{i t-1}-(1-\rho) \mu_{i 2}\right)^{2}+\left(1-\rho^{2}\right)\left(\mu_{i 1}-y_{i 0}\right)^{2} \\
& \left.\left.+\left(1-\rho^{2}\right)\left(\mu_{i 2}-y_{i 0}\right)^{2}\right\}\right] d \mu_{1} d \mu_{2} d \tau d \lambda d \rho \\
= & \int_{a}^{1} \int_{0}^{\infty} \int_{0}^{\infty} \int_{-\infty}^{\infty} \int_{-\infty}^{\infty} \frac{\tau^{\frac{n T}{2}}+n-1}{(2 \pi)^{\frac{n T}{2}+n}\left(1-\rho^{2}\right)^{n}} \exp \left[-\frac{\tau}{2} \sum_{i=1}^{n}\left\{\mu_{i 1}^{2}\left(T_{B}(1-\rho)^{2}+\left(1-\rho^{2}\right)\right)\right.\right. \\
& -2 \mu_{i 1}\left((1-\rho) \sum_{t=1}^{T_{B}}\left(y_{i t}-\rho y_{i t-1}\right)+\left(1-\rho^{2}\right) y_{i 0}\right)+\mu_{i 2}^{2}\left(\frac{T-T_{B}}{\lambda}(1-\rho)^{2}+\left(1-\rho^{2}\right)\right) \\
& -2 \mu_{i 2}\left(\left(\frac{1-\rho}{\lambda}\right) \sum_{t=T_{B}+1}^{T}\left(y_{i t}-\rho y_{i t-1}\right)+\left(1-\rho^{2}\right) y_{i 0}\right)+\sum_{t=1}^{T_{B}}\left(y_{i t}-\rho y_{i t-1}\right)^{2} \\
& \left.\left.\left.+\frac{1}{\lambda} \sum_{t=T_{B}+1}^{T} y_{i t}-\rho y_{i t-1}\right)^{2}+2\left(1-\rho^{2}\right) y_{i 0}^{2}\right\}\right] d \mu_{1} d \mu_{2} d \tau d \lambda d \rho \\
= & \int_{a}^{1} \int_{0}^{\infty} \int_{0}^{\infty} \frac{\tau^{\frac{n T}{2}}-1\left(1-\rho^{2}\right)^{n}}{(2 \pi)^{\frac{n T}{2}}(1-a) \lambda[M(\rho)]^{\frac{n}{2}}[G(\rho, \lambda)]^{\frac{n}{2}}} \exp \left[-\frac{\tau}{2} \sum_{i=1}^{n}\left\{\sum_{t=1}^{T_{B}}\left(y_{i t}-\rho y_{i t-1}\right)^{2}\right.\right. \\
& \left.\left.+\frac{1}{\lambda} \sum_{t=T_{B}+1}^{T}\left(y_{i t}-\rho y_{i t-1}\right)^{2}+2\left(1-\rho^{2}\right) y_{i 0}^{2}-\frac{[K(\rho)]^{2}}{M(\rho)}-\frac{[H(\rho, \lambda)]^{2}}{G(\rho, \lambda)}\right\}\right] d \tau d \lambda d \rho \\
= & \int_{a}^{1} \int_{0}^{\infty} \frac{\left(1-\rho^{2}\right)^{n} \Gamma\left(\frac{n T}{2}\right)}{(\pi)^{\frac{n T}{2}}(1-a) \lambda[M(\rho)]^{\frac{n}{2}}[G(\rho, \lambda)]^{\frac{n}{2}}[I(\rho, \lambda)]^{\frac{n T}{2}} d \lambda d \rho}
\end{aligned}
$$


A2: Posterior probability under $H^{2}$

The likelihood function under unit root hypothesis is given as

$$
L(\tau, \lambda \mid y)=\frac{\tau^{\frac{n T}{2}}}{(2 \pi)^{\frac{n T}{2}}} \exp \left[-\frac{\tau}{2} \sum_{i=1}^{n}\left\{\sum_{t=1}^{T_{B}}\left(y_{i t}-y_{i t-1}\right)^{2}+\frac{1}{\lambda} \sum_{t=T_{B}+1}^{T}\left(y_{i t}-y_{i t-1}\right)^{2}\right\}\right]
$$

Combining it with the prior distributions, the posterior probability is

$$
\begin{aligned}
P\left(y \mid H^{2}\right)= & \int_{0}^{\infty} \int_{0}^{\infty} \frac{\tau^{\frac{n T}{2}}-1}{(2 \pi)^{\frac{n T}{2}} \lambda} \exp \left[-\frac{\tau}{2} \sum_{i=1}^{n}\left\{\sum_{t=1}^{T_{B}}\left(y_{i t}-y_{i t-1}\right)^{2}\right.\right. \\
& \left.\left.+\frac{1}{\lambda} \sum_{t=T_{B}+1}^{T}\left(y_{i t}-y_{i t-1}\right)^{2}\right\}\right] d \tau d \lambda \\
= & \int_{0}^{\infty} \frac{\Gamma\left(\frac{n T}{2}\right)}{(\pi)^{\frac{n T}{2}} \lambda[S(\lambda)]^{\frac{n T}{2}}} d \lambda
\end{aligned}
$$

A3: Posterior probability under $H^{4}$

The likelihood function for the model containing break in variance is given as

$$
\begin{aligned}
L(\tau, \rho, \mu, \lambda \mid y)= & \frac{\tau^{\frac{n T}{2}}}{(2 \pi)^{\frac{n T}{2}}} \exp \left[-\frac{\tau}{2} \sum_{i=1}^{n}\left\{\sum_{t=1}^{T_{B}}\left(y_{i t}-\rho y_{i t-1}-(1-\rho) \mu_{i}\right)^{2}\right.\right. \\
& \left.\left.+\frac{1}{\lambda} \sum_{t=T_{B}+1}^{T}\left(y_{i t}-\rho y_{i t-1}-(1-\rho) \mu_{i}\right)^{2}\right\}\right]
\end{aligned}
$$

The expression of posterior probability can be derive with the help of likelihood function and prior distribution which is given as

$$
\begin{aligned}
P\left(y \mid H^{4}\right)= & \int_{a}^{1} \int_{0}^{\infty} \int_{0}^{\infty} \int_{-\infty}^{\infty} \frac{\tau^{\frac{n T+n}{2}-1}\left(1-\rho^{2}\right)^{\frac{n}{2}}}{(2 \pi)^{\frac{n T+n}{2}}(1-a) \lambda} \exp \left[-\frac{\tau}{2} \sum_{i=1}^{n}\left\{\sum_{t=1}^{T_{B}}\left(y_{i t}-\rho y_{i t-1}-(1-\rho) \mu_{i}\right)^{2}\right.\right. \\
& \left.\left.+\frac{1}{\lambda} \sum_{t=T_{B}+1}^{T}\left(y_{i t}-\rho y_{i t-1}-(1-\rho) \mu_{i}\right)^{2}+\left(1-\rho^{2}\right)\left(\mu_{i}-y_{i 0}\right)^{2}\right\}\right] d \mu d \tau d \lambda d \rho \\
= & \int_{a}^{1} \int_{0}^{\infty} \int_{0}^{\infty} \int_{-\infty}^{\infty} \frac{\tau^{\frac{n T+n}{2}-1}\left(1-\rho^{2}\right)^{\frac{n}{2}}}{(2 \pi)^{\frac{n T+n}{2}}(1-a) \lambda} \exp \left[-\frac{\tau}{2} \sum_{i=1}^{n}\left\{\mu _ { i } ^ { 2 } \left(\left(T_{B}+\frac{1}{\lambda}\left(T-T_{B}\right)\right)(1-\rho)^{2}\right.\right.\right. \\
& \left.+\left(1-\rho^{2}\right)\right)-2 \mu_{i}\left((1-\rho) \sum_{t=1}^{T_{B}}\left(y_{i t}-\rho y_{i t-1}\right)+\left(\frac{1-\rho}{\lambda}\right) \sum_{t=T_{B}+1}^{T}\left(y_{i t}-\rho y_{i t-1}\right)\right. \\
& \left.+\left(1-\rho^{2}\right) y_{i 0}\right)+\sum_{t=1}^{T_{B}}\left(y_{i t}-\rho y_{i t-1}\right)^{2}+\frac{1}{\lambda} \sum_{t=T_{B}+1}^{T}\left(y_{i t}-\rho y_{i t-1}\right)^{2} \\
& \left.\left.+\left(1-\rho^{2}\right) y_{i 0}^{2}\right\}\right] d \mu d \tau d \lambda d \rho \\
= & \int_{a}^{1} \int_{0}^{\infty} \int_{0}^{\infty} \frac{\tau^{\frac{n T}{2}-1}\left(1-\rho^{2}\right)^{\frac{n}{2}}}{(2 \pi)^{\frac{n T}{2}}(1-a) \lambda[A(\rho, \lambda)]^{\frac{n}{2}}} \exp \left[-\frac{\tau}{2} \sum_{i=1}^{n}\left\{\sum_{t=1}^{T_{B}}\left(y_{i t}-\rho y_{i t-1}\right)^{2}\right.\right. \\
& \left.\left.+\frac{1}{\lambda} \sum_{t=T_{B}+1}^{T}\left(y_{i t}-\rho y_{i t-1}\right)^{2}+\left(1-\rho^{2}\right) y_{i 0}^{2}-\frac{[B(\rho, \lambda)]^{2}}{A(\rho, \lambda)}\right\}\right] d \tau d \lambda d \rho \\
= & \int_{a}^{1} \int_{0}^{\infty} \frac{\left(1-\rho^{2}\right)^{\frac{n}{2}} \Gamma\left(\frac{n T}{2}\right)}{(\pi)^{\frac{n T}{2}}(1-a) \lambda[A(\rho, \lambda)]^{\frac{n}{2}}[C(\rho, \lambda)]^{\frac{n T}{2}} d \lambda d \rho}
\end{aligned}
$$


A4: Posterior probability under $H^{5}$

The likelihood function is given under this hypothesis is

$$
\begin{aligned}
L\left(\tau, \rho, \mu_{1}, \mu_{2} \mid y\right)= & \frac{\tau^{\frac{n T}{2}}}{(2 \pi)^{\frac{n T}{2}}} \exp \left[-\frac{\tau}{2} \sum_{i=1}^{n}\left\{\sum_{t=1}^{T_{B}}\left(y_{i t}-\rho y_{i t-1}-(1-\rho) \mu_{i 1}\right)^{2}\right.\right. \\
& \left.\left.+\sum_{t=T_{B}+1}^{T}\left(y_{i t}-\rho y_{i t-1}-(1-\rho) \mu_{i 2}\right)^{2}\right\}\right]
\end{aligned}
$$

The posterior probability is found by

$$
\begin{aligned}
& P\left(y \mid H^{5}\right)= \int_{a}^{1} \int_{0}^{\infty} \int_{-\infty}^{\infty} \int_{-\infty}^{\infty} \frac{\tau^{\frac{n T}{2}+n-1}\left(1-\rho^{2}\right)^{n}}{(2 \pi)^{\frac{n T}{2}+n}(1-a)} \exp \left[-\frac{\tau}{2} \sum_{i=1}^{n}\left\{\sum_{t=1}^{T_{B}}\left(y_{i t}-\rho y_{i t-1}-(1-\rho) \mu_{i 1}\right)^{2}\right.\right. \\
&+\sum_{t=T_{B}+1}^{T}\left(y_{i t}-\rho y_{i t-1}-(1-\rho) \mu_{i 2}\right)^{2}+\left(1-\rho^{2}\right)\left(\mu_{i 1}-y_{i 0}\right)^{2} \\
&\left.\left.+\left(1-\rho^{2}\right)\left(\mu_{i 2}-y_{i 0}\right)^{2}\right\}\right] d \mu_{1} d \mu_{2} d \tau d \rho \\
&= \int_{a}^{1} \int_{0}^{\infty} \int_{-\infty}^{\infty} \int_{-\infty}^{\infty} \frac{\tau^{\frac{n T}{2}+n-1}\left(1-\rho^{2}\right)^{n}}{(2 \pi)^{\frac{n T}{2}+n}(1-a)} \exp \left[-\frac{\tau}{2} \sum_{i=1}^{n}\left\{\mu_{i 1}^{2}\left(T_{B}(1-\rho)^{2}+\left(1-\rho^{2}\right)\right)\right.\right. \\
&-2 \mu_{i 1}\left((1-\rho) \sum_{t=1}^{T_{B}}\left(y_{i t}-\rho y_{i t-1}\right)+\left(1-\rho^{2}\right) y_{i 0}\right)+\sum_{t=1}^{T_{B}}\left(y_{i t}-\rho y_{i t-1}\right)^{2} \\
&+\sum_{t=T_{B}+1}^{T}\left(y_{i t}-\rho y_{i t-1}\right)^{2}-2 \mu_{i 2}\left((1-\rho) \sum_{t=T_{B}+1}^{T}\left(y_{i t}-\rho y_{i t-1}\right)+\left(1-\rho^{2}\right) y_{i 0}\right) \\
&\left.\left.+\mu_{i 2}^{2}\left(\left(T-T_{B}\right)(1-\rho)^{2}+\left(1-\rho^{2}\right)\right)+2\left(1-\rho^{2}\right) y_{i 0}^{2}\right\}\right] d \mu_{1} d \mu_{2} d \tau d \rho \\
&=\int_{a}^{1} \int_{0}^{\infty} \frac{\tau^{\frac{n T}{2}-1}\left(1-\rho^{2}\right)^{n}}{(2 \pi)^{\frac{n T}{2}}(1-a)[M(\rho)]^{\frac{n}{2}}[N(\rho)]^{\frac{n}{2}}} \exp \left[-\frac{\tau}{2} \sum_{i=1}^{n}\left\{\sum_{t=1}^{T}\left(y_{i t}-\rho y_{i t-1}\right)^{2}\right.\right. \\
&\left.\left.\quad+2\left(1-\rho^{2}\right) y_{i 0}^{2}-\frac{[K(\rho)]^{2}}{M(\rho)}-\frac{[L(\rho)]^{2}}{N(\rho)}\right\}\right] d \tau d \rho \\
&=\int_{a}^{1} \frac{\left(1-\rho^{2}\right)^{n} \Gamma\left(\frac{n T}{2}\right)}{(\pi)^{\frac{n T}{2}}(1-a) \lambda[M(\rho)]^{\frac{n}{2}}[N(\rho)]^{\frac{n}{2}}[O(\rho)]^{\frac{n T}{2}}} d \rho
\end{aligned}
$$

A5: Posterior probability under $H^{6}$

No break is consider under unit root hypothesis, the likelihood function is given as

$$
L(\tau \mid y)=\frac{\tau^{\frac{n T}{2}}}{(2 \pi)^{\frac{n T}{2}}} \exp \left[-\frac{\tau}{2} \sum_{i=1}^{n} \sum_{t=1}^{T}\left(y_{i t}-y_{i t-1}\right)^{2}\right]
$$

Combining the prior distributions, the posterior probability is obtained

$$
\begin{aligned}
P\left(y \mid H^{6}\right) & =\int_{0}^{\infty} \frac{\tau^{\frac{n T}{2}}-1}{(2 \pi)^{\frac{n T}{2}}} \exp \left[-\frac{\tau}{2} \sum_{i=1}^{n} \sum_{t=1}^{T}\left(y_{i t}-y_{i t-1}\right)^{2}\right] d \tau \\
& =\frac{\Gamma\left(\frac{n T}{2}\right)}{(\pi)^{\frac{n T}{2}} \lambda\left[\sum_{i=1}^{n} \sum_{t=1}^{T}\left(y_{i t}-y_{i t-1}\right)^{2}\right]^{\frac{n T}{2}}} d \lambda
\end{aligned}
$$

A6: Posterior probability under $H^{8}$

The likelihood function is given as

$$
L(\tau, \rho, \mu \mid y)=\frac{\tau^{\frac{n T}{2}}}{(2 \pi)^{\frac{n T}{2}}} \exp \left[-\frac{\tau}{2} \sum_{i=1}^{n} \sum_{t=1}^{T}\left(y_{i t}-\rho y_{i t-1}-(1-\rho) \mu_{i}\right)^{2}\right]
$$


The posterior probability is

$$
\begin{aligned}
P\left(y \mid H^{8}\right)= & \int_{a}^{1} \int_{0}^{\infty} \int_{-\infty}^{\infty} \frac{\tau^{\frac{n T+n}{2}-1}\left(1-\rho^{2}\right)^{\frac{n}{2}}}{(2 \pi)^{\frac{n T+n}{2}}(1-a)} \exp \left[-\frac{\tau}{2} \sum_{i=1}^{n}\left\{\sum_{t=1}^{T}\left(y_{i t}-\rho y_{i t-1}-(1-\rho) \mu_{i}\right)^{2}\right.\right. \\
& \left.\left.+\left(1-\rho^{2}\right)\left(\mu_{i}-y_{i 0}\right)^{2}\right\}\right] d \mu d \tau d \rho \\
= & \int_{a}^{1} \int_{0}^{\infty} \int_{-\infty}^{\infty} \frac{\tau^{\frac{n T+n}{2}-1}\left(1-\rho^{2}\right)^{\frac{n}{2}}}{(2 \pi)^{\frac{n T+n}{2}}(1-a)} \exp \left[-\frac{\tau}{2} \sum_{i=1}^{n}\left\{\mu_{i}^{2}\left(T(1-\rho)^{2}+\left(1-\rho^{2}\right)\right)+\left(1-\rho^{2}\right) y_{i 0}^{2}\right.\right. \\
& \left.\left.-2 \mu_{i}\left((1-\rho) \sum_{t=1}^{T}\left(y_{i t}-\rho y_{i t-1}\right)+\left(1-\rho^{2}\right) y_{i 0}\right)+\sum_{t=1}^{T}\left(y_{i t}-\rho y_{i t-1}\right)^{2}\right\}\right] d \mu d \tau d \rho \\
= & \int_{a}^{1} \int_{0}^{\infty} \frac{\tau^{\frac{n T}{2}}-1\left(1-\rho^{2}\right)^{\frac{n}{2}}}{(2 \pi)^{\frac{n T}{2}}(1-a)[P(\rho)]^{\frac{n}{2}}} \exp \left[-\frac{\tau}{2} \sum_{i=1}^{n}\left\{\sum_{t=1}^{T}\left(y_{i t}-\rho y_{i t-1}\right)^{2}\right.\right. \\
& \left.\left.+\left(1-\rho^{2}\right) y_{i 0}^{2}-\frac{[Q(\rho)]^{2}}{P(\rho)}\right\}\right] d \tau d \rho \\
P= & \int_{a}^{1} \frac{\left(1-\rho^{2}\right)^{\frac{n}{2}} \Gamma\left(\frac{n T}{2}\right)}{(\pi)^{\frac{n T}{2}}(1-a) \lambda[(\rho)]^{\frac{n}{2}}[R(\rho)]^{\frac{n T}{2}}} d \rho
\end{aligned}
$$

Utilizing (A1) to (A6), we obtain the expression for required Theorem 4.1 to 4.6. 